\title{
Algebraic Parameter Identification of Nonlinear Vibrating Systems and Non Linearity Quantification Using the Hilbert Transformation
}

\author{
Luis Gerardo Trujillo-Franco $\left(\mathbb{D},{ }^{1}\right.$ Gerardo Silva-Navarro $\left(\mathbb{D},{ }^{2}\right.$ \\ and Francisco Beltran-Carbajal $\mathbb{D}^{3}$ \\ ${ }^{1}$ Universidad Politecnica de Pachuca, Automotive Mechanical Engineering, Zempoala, Hidalgo, Mexico \\ ${ }^{2}$ Centro de Investigacion y de Estudios Avanzados del IPN, Departamento de Ingenieria Electrica, Seccion de Mecatronica, \\ Mexico City, Mexico \\ ${ }^{3}$ Universidad Autónoma Metropolitana, Unidad Azcapotzalco, Departamento de Energía, Mexico City, Mexico
}

Correspondence should be addressed to Francisco Beltran-Carbajal; fbeltran@azc.uam.mx

Received 19 February 2021; Revised 2 June 2021; Accepted 14 June 2021; Published 23 June 2021

Academic Editor: Libor Pekař

Copyright (c) 2021 Luis Gerardo Trujillo-Franco et al. This is an open access article distributed under the Creative Commons Attribution License, which permits unrestricted use, distribution, and reproduction in any medium, provided the original work is properly cited.

\begin{abstract}
A novel algebraic scheme for parameters' identification of a class of nonlinear vibrating mechanical systems is introduced. A nonlinearity index based on the Hilbert transformation is applied as an effective criterion to determine whether the system is dominantly linear or nonlinear for a specific operating condition. The online algebraic identification is then performed to compute parameters of mass and damping, as well as linear and nonlinear stiffness. The proposed algebraic parametric identification techniques are based on operational calculus of Mikusiński and differential algebra. In addition, we propose the combination of the introduced algebraic approach with signals approximation via orthogonal functions to get a suitable technique to be applied in embedded systems, as a digital signals' processing routine based on matrix operations. A satisfactory dynamic performance of the proposed approach is proved and validated by experimental case studies to estimate significant parameters on the mechanical systems. The presented online identification approach can be extended to estimate parameters for a wide class of nonlinear oscillating electric systems that can be mathematically modelled by the Duffing equation.
\end{abstract}

\section{Introduction}

Accurate fast parameter identification of vibrating mechanical systems constitutes an active research subject. Optimization algorithms, least squares, time series, statistical methods, spectral analysis, Volterra series, wavelets, and orthogonal functions have been used for development of parametric identification techniques [1-3]. In [4], optimization techniques, combined with classic control theory, have been introduced for system parameters' identification, and a special application for parameters' identification for active vibration absorption schemes is reported in [5] where the offline modal analysis are implemented in the presence of noise. A model for linear nonviscous damping and a time- domain method for the identification of this parameter is proposed in [6]. In the context of nonlinear systems, a black box system identification technique based on co-evolutionary algorithms and neural networks is proposed in [7], and this approach is applied to a magnetorheological damper. As a process, system parameters' identification involves a sequence of systematic stages. The final of those stages involves the application of special tools such as specialized software that features several numerical methods for processing and analyzing the signals obtained from experimental tests applied to mechanical systems under the study. All of these efforts are conducted for achieving the final goal of building a mathematical model for the description of the dynamic behavior of a specific vibrating 
mechanical system. Standard mathematical tools for identification purposes are methods used to analyze considerably large amounts of experimental data. As a result of this numerical analysis, the mathematical model of the system dynamics behavior is then built in terms of the determined or identified parameters. Classical mathematical modelling of vibrating systems is commonly based on linear assumptions on their dynamical behavior. In this way, it is possible to use basic and well-behaved approaches such as least squares and autoregressive models [3, 8-10]. Nevertheless, in modern materials and structural engineering, large displacements, geometrical restrictions, and complex behavior are now becoming common in modern mechanical structures resulting on inherent nonlinear phenomena. Hence, despite of numerous advantages of linearity assumptions on mechanical systems, there are cases where linear methods are not longer effective or even valid $[3,11,12]$.

Nowadays, evident developments in computing sciences and great capabilities of modern and multitasking microprocessors and microcontrollers [13-15] open the doors to the possibility of applying novel and sophisticated numerical methods, which allow to perform interesting and before unacceptably, complicated online parameters' identification schemes for adaptive control [16]. Thus, complex problems, such as nonlinearities in mechanical systems, as that reported in [17], can be addressed by using mathematical tools, which in the past were purely theoretical and very hard to prove with experimental data, for the practical application of diverse nonlinear systems' identification schemes as the ones reported in [18-20], where the identification of the state equation in nonlinear systems is presented for two interesting simulation cases. These approaches are based on system signals' approximation by determining an analytical function $\hat{g}$ that approximates the actual (unknown) system state equation $g$, with the form of $\hat{g}$ including suitable basis functions that are relevant to the specific problem. Certainly, there are challenges and limitations in the use of microcontrollers in the context of strict real-time applications, given the inherent nature of their reduced instruction set architecture, called RISC, mainly in quadratic programming applications for optimization. However, in the present work, the use of these advanced digital systems in an algebraic identification scheme is proposed with satisfactory results.

In this work, we present an online algebraic identification method based on the important mathematical tools of Mikusiński's operational calculus, orthogonal functions' signal approximation, and application of Hilbert transforms, to compute the main physical parameters of a vibrating mechanical system, using measurements of its response under the action of exogenous forces. We use Hilbert transforms as an indicator of presence of nonlinearities, by using the properties of this linear transformation as reported in $[8,12]$. On the contrary, we apply an algebraic approach to transform a complex calculus problem into an algebraic equation [21] in terms of the parameters to be identified; this equation has an iterated time integral structure, such that we can take advantage of the orthogonal functions signal approximation in order to make compact and easier to perform iterated integrals $[22,23]$. Analytical and experimental results are described to prove the effectiveness of the proposed algebraic scheme for online parameter estimation of the nonlinear vibrating system. The proposed algebraic identification scheme can be directly extended to estimate parameters for a wide class of nonlinear oscillating electric systems that can be mathematically modelled by the Duffing equation [24]. The main contributions of the present work are summarized as follows:

(i) An algebraic method for online and time-domain identification of parameters for an important class of nonlinear vibrating systems is presented and evaluated in several experimental case studies

(ii) The proposed algebraic estimation approach requires a small interval of time to provide accurate results

(iii) Compared to other parameter identification methods, a significant reduction of the amount of data required for the estimation process is an important highlight

(iv) The approximation of signals by means of orthogonal polynomials, in combination with the algebraic approach, provides robustness and simplifies the computation of iterated integrations

This paper is organized as follows. The class of nonlinear vibrating mechanical system considered for algebraic and online parameters estimation is described in Section 2. In addition, a nonlinearity detection method, based on the Hilbert transformation, is presented. The experimental verification of the proposed identification scheme is described in Section 3, where the performance of the algebraic identification approach is evaluated in two case studies. The nonlinearity detection method described in Section 2 is verified on both of the case studies. A combination of the algebraic estimation technique with the signals approximation using orthogonal polynomials is described in Section 4. The resulting technique represents an alternative to implement estimations of system parameters using buffered signals. Finally, main conclusions of the present study are described in Section 5.

\section{Nonlinear Vibrating System}

2.1. Mathematical Model of the Nonlinear Vibrating System. Consider the vibrating mechanical system shown in Figure 1. The inherent dynamic behavior of the vibrating mechanical system is determined by the parameters of mass $m_{i}$ and nonlinear coupling elements that produce the forces $F_{s i}$ and $F_{d i}[22,24]$. Those nonlinear functions of displacements and velocities, $x_{i}$ and $\dot{x}_{i}$, describe the nonlinear stiffness and nonlinear damping effects, respectively, and are defined as follows: where $b_{i}$ denotes viscous damping and $f_{c i}$ stands for the Coulomb friction coefficient, and the 


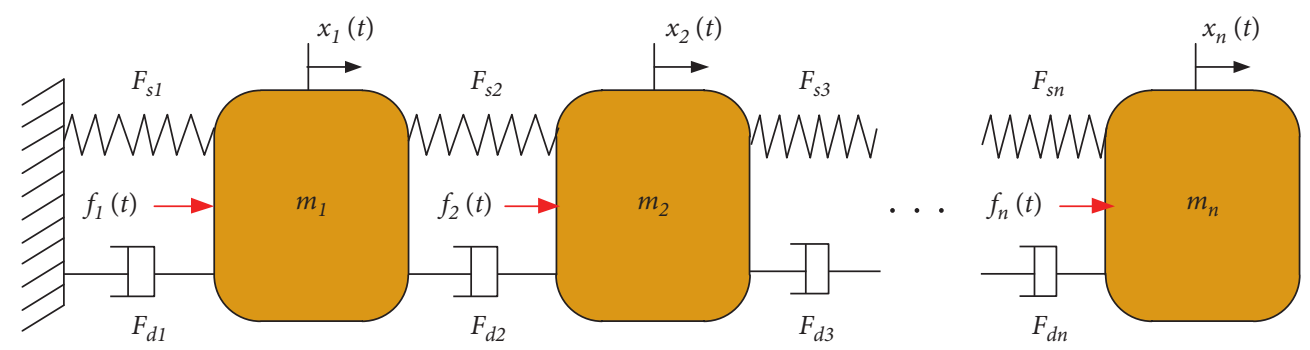

FIgURE 1: Schematic diagram of a general nonlinear mechanical system.

constants $k_{i j}, j=1,2, \ldots, r$, with $r$ a positive integer, represent polynomial stiffness coefficients. The function $\operatorname{sgn}(\dot{x})$ is defined by

$$
\begin{aligned}
& F_{s i}= k_{i 1}\left(x_{i}-x_{i-1}\right)+k_{i 2}\left(x_{i}-x_{i-1}\right)^{2} \\
&+\cdots+k_{i r}\left(x_{i}-x_{i-1}\right)^{r} \\
&= \sum_{j=1}^{r} k_{i j}\left(x_{i}-x_{i-1}\right)^{j}, \\
& F_{d i}= b_{i}\left(\dot{x}_{i}-\dot{x}_{i-1}\right)+f_{c i} \operatorname{sgn}\left(\dot{x}_{i}\right), \\
& i= 1,2, \ldots, n, x_{0} \equiv 0, \\
& \operatorname{sgn}(\dot{x})=\left\{\begin{array}{cc}
1, & \text { if } \dot{x} \geq 0, \\
-1, & \text { if } \dot{x}<0
\end{array}\right\} .
\end{aligned}
$$

For each degree of freedom associated with the position coordinate $x_{i}$, the nonlinear system dynamics can be described by the set of coupled differential equations:

$$
\begin{aligned}
m_{i} \ddot{x}_{i} & +F_{s i}+F_{d i}+b_{i+1}\left(\dot{x}_{i}-\dot{x}_{i+1}\right) \\
& -\sum_{j=1}^{r} k_{i+1 j}\left(x_{i+1}-x_{i}\right)^{j}=f_{i}, \quad i=1,2, \ldots, n, x_{n+1} \equiv 0 .
\end{aligned}
$$

Thus, we can express the dynamic behavior of the nonlinear system shown in Figure 1 in the matrix form

$$
\mathbf{M} \ddot{x}+\mathbf{B} \dot{x}+\mathbf{K} \mathbf{x}+Q \underline{Q}(\mathbf{y}, \dot{x})=\mathbf{f}(t),
$$

where the vector $\mathbf{x} \in R^{n}$ denotes the physical displacements of the masses as a function of time $t$ and the relative displacements $\quad \mathbf{y}=\left[\left(x_{2}-x_{1}\right),\left(x_{3}-x_{2}\right), \ldots,\left(x_{n+1}-x_{n}\right)\right]^{T}$, $\mathbf{f} \in R^{n}$ is an exogenous force vector, and the function $Q(\mathbf{y}, \dot{x}) \in R^{n}$ is a nonlinear restoring force, commonly depending on the displacements and velocities of the $n$ degrees of freedom. The dynamic response of the linear part is determined for the mass, linear damping, and stiffness matrices: $\mathbf{M} \in R^{n \times n}, \mathbf{B} \in R^{n \times n}$, and $\mathbf{K} \in R^{n \times n}$. The nonlinear restoring force takes a structure such that

$$
Q(\mathbf{y}, \dot{\mathbf{x}})=\mathbf{K}_{2} \mathbf{y}^{2}+\mathbf{K}_{3} \mathbf{y}^{3}+\cdots+\mathbf{K}_{r} \mathbf{y}^{r}+\mathbf{F}_{c} \operatorname{sgn}(\dot{\mathbf{x}}),
$$

where $\mathbf{K}_{\mathbf{j}} \in R^{n \times n}$ with $j=2,3, \ldots, r$ are polynomic stiffness matrices and $\mathbf{F}_{\mathbf{c}} \in R^{n \times n}$ is the Coulomb friction matrix. Equation (5) implies a piecewise operation such that $\mathbf{y}^{r}=\left[\left(x_{2}-x_{1}\right)^{r},\left(x_{3}-x_{2}\right)^{r}, \ldots,\left(x_{n+1}-x_{n}\right)^{r}\right]^{T}$. Now, it is important and necessary to have an indicator of how important or dominant are the nonlinear terms over the global dynamic response of the mechanical system. In the next section, we present the application of a mathematical method for determining this influence in terms of a numerical indicator.

2.2. Nonlinearity Detection. There exist numerous methods for determining the influence of nonlinearities present in the system dynamics $[12,25,26]$. When assuming linear behavior on the system, it is possible to use basic approaches such as least squares and autoregressive models for control purposes $[3,27]$. Despite of the numerous advantages of the linearity assumption on mechanical systems, there are cases where the linear methods are ineffective or inoperative. It is well known that the use of the Hilbert transform in the analysis of nonlinear systems is a well-founded tool $[8,12]$. The Hilbert transform pairs, as described in [8], of an specific frequency response function $F(\omega)$, also known as system FRF, are defined as

$$
\begin{aligned}
& \operatorname{Re}(F(\omega))=-\frac{1}{\pi} c p \int_{-\infty}^{\infty} \frac{\operatorname{Im}(F(\omega))}{\omega-\omega_{c}} \mathrm{~d} \omega=H\{\operatorname{Im}(F(\omega))\} \\
& \operatorname{Im}(F(\omega))=\frac{1}{\pi} c p \int_{-\infty}^{\infty} \frac{\operatorname{Re}(F(\omega))}{\omega-\omega_{c}} \mathrm{~d} \omega=-H\{\operatorname{Re}(F(\omega))\}
\end{aligned}
$$

where $H\{\}$ denotes the Hilbert transformation operator. The terms $\operatorname{Re}(F(\omega))$ and $\operatorname{Im}(F(\omega))$ denote the real and imaginary part of the complex function $F(\omega)$, respectively. The constant $c p$ denotes the Cauchy principal value of the integral, used by the singularity at $\omega=\omega_{c}$ into the integrand. Relations defined by (6) and (7) are not valid for nonlinear systems, and, as a consequence, the Hilbert transformation $H\{F(\omega)\}$ results in a distorted version of the original $F(\omega)$. This distortion is then used as a nonlinearity indicator, numerically quantifiable, that determines the level of nonlinear behavior of the system under analysis. The cross correlation coefficient is a numerical index used for this purpose:

$$
\eta_{H i}=\left\|X_{H F}(0)\right\|^{2}
$$

where $\left\|X_{H F}(0)\right\|$ is the normalized cross correlation coefficient defined by 


$$
X_{H F}(\Delta \omega)=\int_{-\infty}^{\infty} H(\omega) F(\omega+\Delta \omega) \mathrm{d} \omega
$$

where $F(\omega)$ is the FRF of the system and $H(\omega)$ the Hilbert transform of $F(\omega)$. The numerical value $\eta_{H i}$ indicates nonlinearity in the system at a specific input amplitude. In this work, we use this index to study the presence of nonlinearities in the system under analysis, where, for a linear system, the expected value of $\eta_{H i}$ is precisely 1 . Here, we consider a particular linearity criterion, as reported in [8]. Thus, we can consider a value of $0.9 \leq \eta \leq 1$ for a linearity assumption of the system. Values under 1 are considered as a clear indicative of nonlinear behavior of the system.

Remark 1. There are significant advances and improvements on the application of the Hilbert Transformation for the time-domain identification of the instantaneous frequency and damping ratios $[8,18]$. Those developments and tools suggest the use of them for the implementation of linear and nonlinear systems' parameter estimation. However, we consider important to make clear to the reader that we are not using the Hilbert transformation-based methods to identify systems' parameters. We use the Hilbert transformation pairs (7) as a mathematical tool for the quantification of the nonlinear behavior of the system by analyzing its FRF.

\section{Experimental Verification}

3.1. First Case Study: One-Degree-of-Freedom Nonlinear Vibrating System. The experimental setup shown in Figure 2 is a configuration for a nonlinear vibrating system of one degree of freedom, where its corresponding schematic diagram is also depicted. The mechanical system consists of a mass carriage, attached to a nonlinear spring. The mass carriage has an antifriction ball bearing system, the mass carriage has a (rotary) high-resolution optical encoder to measure its actual position via cable-pulley system, where the effective resolution is 2266 pulses $/ \mathrm{cm}$.

The nonlinear spring shown in detail in Figure 3 presents a polynomial restoring behavior $F(\delta)=k_{p} \delta^{3}+k \delta$, which is described by the experimental data chart, also shown in Figure 3. The numeric values of $k_{p}$ and $k$ were determined by applying a least squares curve fitting method to the experimental data, where their corresponding magnitudes are reported in Table 1.

The degree of freedom under analysis consists of one mass carriage connected to a fixed support by the nonlinear rubber elastic element described before. The mass carriage suspension has antifriction ball bearing systems such that we can neglect the dry friction. The mass carriage has a (rotary) high-resolution optical encoder to measure its actual position $x(t)$ via a cable-pulley system. The nonlinear differential equation that describes this dynamical system is given by

$$
m \ddot{x}+b \dot{x}+q(x, \dot{x})=f(t)
$$

with

$$
q(x, \dot{x})=k x+k_{p} x^{3}
$$

3.2. Frequency Response Function (FRF) and Nonlinearity Index Calculation. The frequency analysis of the nonlinear mechanical system shown in Figure 4 was conducted by applying a harmonic sinusoidal swept $f(t)=A \sin [\omega(t) t]$, with a constant amplitude of $A=2.96 \mathrm{~N}$ and a time-varying frequency $\omega(t)=1.25 t \mathrm{~Hz}$. The time-domain chart and the corresponding system response to the sinusoidal swept is shown in Figure 5.

The corresponding FRF is reported in Figure 4 where it is possible to observe a clear distortion on the Hilbert transform of the original FRF (on blue) at this particular amplitude of the input force, which is evident in the Argand (Real, Imag) chart, as depicted in Figure 4.

In order to evaluate the effects of the amplitude on the distortion produced by the Hilbert transformation over the original FRF, a set of sinusoidal sweeps, similar with the same frequency range and several different amplitudes were performed to the system. The effect of the amplitude on the nonlinearity index calculated according to equation (8) is reported in Figure 6. It is clear that the nonlinear effects are specially evident at amplitudes bigger than $2 \mathrm{~N}$.

In previous works, time-domain system parameters' identification has been proposed and verified in experiments and numerical simulations [28, 29], which involves the use of operational calculus for the algebraic manipulation of differential equations (see [21]). The proposed identification scheme is robust and effective for both linear and nonlinear systems. In addition, the system parameters are estimated in a time-domain and online fashion by using measurements of the system input and output. In this work, we present experimental results of the evaluation of the algebraic approach on a particular experimental setup with geometric nonlinearities. For synthesis of online and time-domain parameter estimators, equation (10) is multiplied by $(\Delta t)^{2}=$ $\left(t-t_{0}\right)^{2}$ and then integrated by parts with respect to time yielding:

$$
\begin{aligned}
& m\left[2 \int_{t_{0}}^{(2)} x-4 \int_{t_{0}}(\Delta t) x+(\Delta t)^{2} x\right] \\
& +b\left[-2 \int_{t_{0}}^{(2)}(\Delta t) x+\int_{t_{0}}(\Delta t)^{2} x\right] \\
& +k \int_{t_{0}}^{(2)}(\Delta t)^{2} x+k_{p} \int_{t_{0}}^{(2)}(\Delta t)^{2} x^{3}=\int_{t_{0}}^{(2)}(\Delta t)^{2} f(t),
\end{aligned}
$$

where $\int_{0}^{(n)} \phi(t)$ is used to denote iterated time integrals of the form:

$$
\int_{t_{0}}^{t} \int_{t_{0}}^{\alpha_{1}} \ldots \int_{t_{0}}^{\alpha_{n-1}} \phi\left(\alpha_{n}\right) d \alpha_{n} \ldots d \alpha_{1} .
$$

Notice that this expression does not depend on the system initial conditions of any involved function. Here, we have an expression for the system parameters $m, b, k$, and $k_{p}$. Notice that the system parameters appear algebraically in 


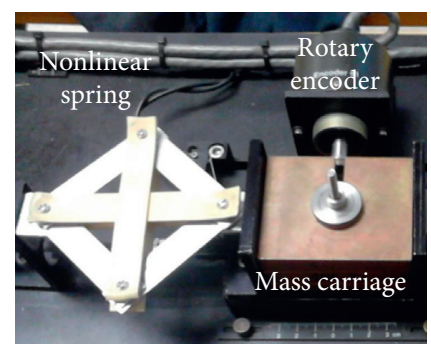

(a)

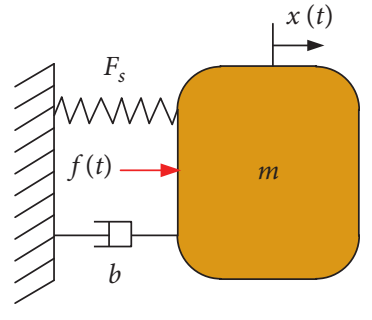

(b)

Figure 2: One degree-of-freedom nonlinear vibrating mechanical system and its corresponding schematic representation.

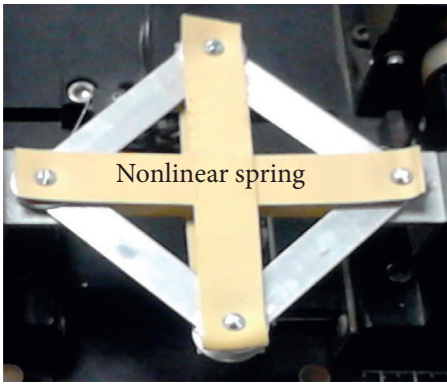

(a)

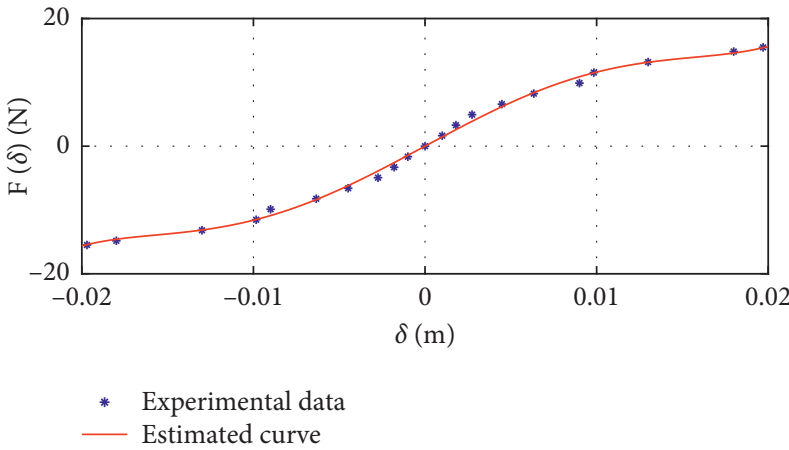

(b)

FIGURE 3: Nonlinear spring and its corresponding curve of force.

TABLE 1: System parameters.

\begin{tabular}{lc}
\hline Parameters & Value \\
\hline$m$ & $2.53 \mathrm{~kg}$ \\
$b$ & $0 \mathrm{Ns} / \mathrm{m}$ \\
$k$ & $1272.1 \mathrm{~N} / \mathrm{m}$ \\
$k_{p}$ & $-1.237 \times 10^{6} \mathrm{~N} / \mathrm{m}^{3}$ \\
\hline
\end{tabular}

equation (12). The identification of the system parameters is achieved by the algebraic manipulation of equation (12) in order to express those parameters by a system of linear equations, whose solution is precisely the set of unknown terms [21, 29]. Hence,

$$
\mathbf{A} \theta=\mathbf{D}
$$

where $\theta=\left[\widehat{m}, \widehat{b}, \widehat{k}, \widehat{k}_{p}\right]$ is the vector of the estimated parameters, $\mathbf{A}$ and $\mathbf{D}$ are, respectively, $4 \times 4$ and $4 \times 1$ matrices given by

$$
\begin{aligned}
& \mathbf{A}=\left[\begin{array}{cccc}
a_{11} & a_{12} & \ldots & a_{14} \\
a_{21} & a_{22} & \ldots & a_{24} \\
\vdots & \vdots & \vdots & \\
a_{41} & a_{42} & \ldots & a_{44}
\end{array}\right], \\
& \mathbf{D}=\left[\begin{array}{c}
d_{1} \\
d_{2} \\
\vdots \\
d_{4}
\end{array}\right] .
\end{aligned}
$$

The components $a_{i, j}$ and $d_{i}$ are 


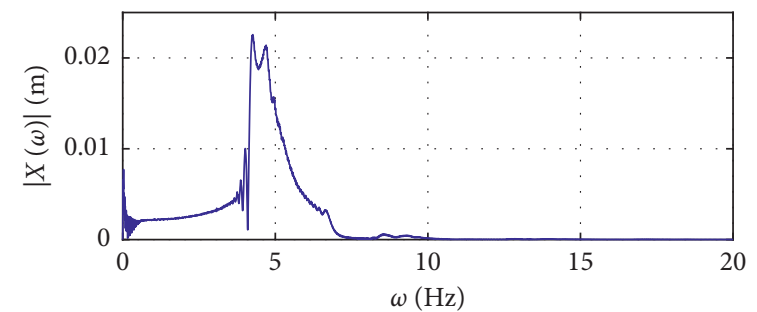

(a)

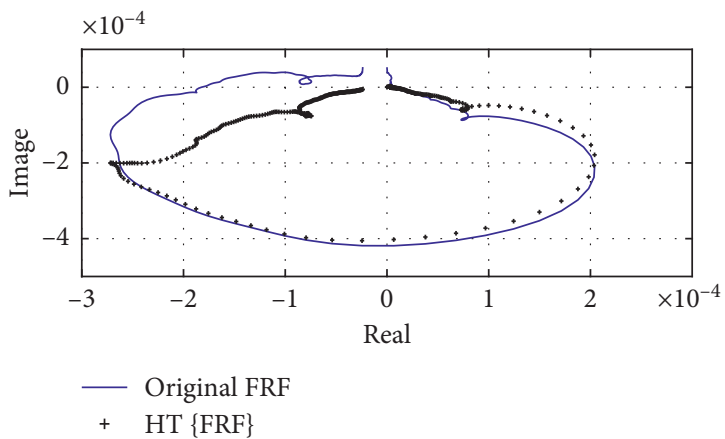

(b)

FIgURE 4: FRF of the system and its Hilbert transformation.

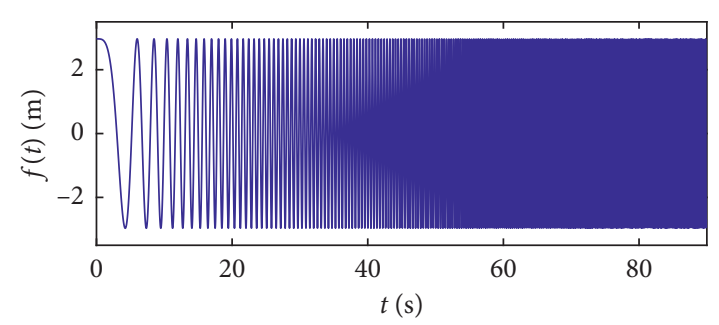

(a)

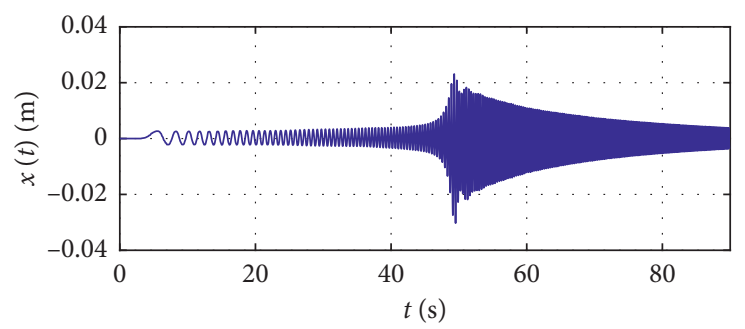

(b)

FIGURE 5: System response under sinusoidal swept excitation.

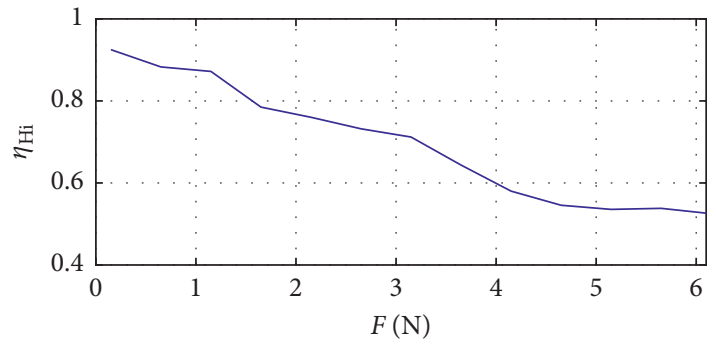

(a)

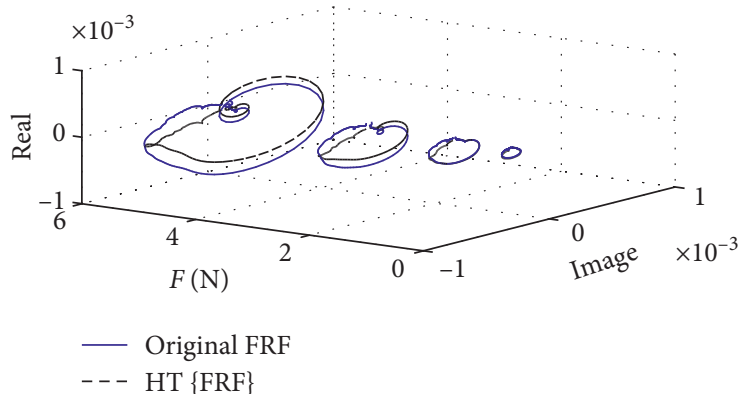

(b)

FIgURE 6: Effect of the amplitude on the nonlinearity index. 


$$
\begin{aligned}
& a_{11}=2 \int_{t_{0}}^{(2)} x-4 \int_{t_{0}}(\Delta t) x+(\Delta t)^{2} x, \\
& a_{12}=-2 \int_{t_{0}}^{(2)}(\Delta t) x+\int_{t_{0}}(\Delta t)^{2} x, \\
& a_{13}=\int_{t_{0}}^{(2)}(\Delta t)^{2} x, \\
& a_{14}=\int_{t_{0}}^{(2)}(\Delta t)^{2} x^{3}, \\
& d_{1}=\int_{t_{0}}^{(2)}(\Delta t)^{2} f .
\end{aligned}
$$

The iterated integrations of equation (16) lead to the rest of the entries or components of the matrices $\mathbf{A}$ and $\mathbf{D}$ as follows:

$$
\begin{aligned}
a_{k j} & =\int_{t_{0}} a_{k-1 j}, \\
d_{k} & =\int_{t_{0}} d_{k-1},
\end{aligned}
$$

with $k=2, \ldots, 4$ and $j=1, \ldots, 4$. Hence,

$$
\theta=(\mathbf{A})^{-1} \mathbf{D}=\frac{1}{\Delta}\left[\begin{array}{c}
\Delta_{1} \\
\vdots \\
\Delta_{4}
\end{array}\right] .
$$

Then, the estimations of the system parameters $\widehat{m}, \widehat{b}, \widehat{k}$, and $\widehat{k}_{p}$ contained as components of the vector $\theta$ can be algebraically computed in some short window of time and without singularities by the estimators:

$$
\hat{\theta}[i]=\operatorname{sgn}\left(\Delta_{i}\right) \operatorname{sgn}(\Delta) \frac{\int_{t_{0}}^{(2)} e^{-\gamma\left(t-t_{0}\right)}\left|\Delta_{i}\right|}{\int_{t_{0}}^{(2)} e^{-\gamma\left(t-t_{0}\right)}|\Delta|}, \quad i=1, \ldots, 4,
$$

where $\gamma \geq 0$ is an invariant filtering and smoothing gain, as used in [29, 30]. Here, - denotes estimate and "sgn" is a function defined in 2 which is only used to get the sign of the nonlinear stiffness parameter $k_{p}$.

For the experimental verification of the algebraic identification scheme, we use a step excitation force with an amplitude of $14 \mathrm{~N}$ and take measurements of the position of the mass carriage at a constant sampling period of $1 \mathrm{~ms}$; both, the position signal $x(t)$ and algebraic identification are obtained through a high-speed DSP board into a standard PC running under Windows $10^{\circledR}$ and Matlab ${ }^{\circledR} /$ Simulink ${ }^{\circledR}$. The parameters of the mechanical system are reported in Table 1 . The excitation force $f(t)$ and the system response $x(t)$ are shown in Figure 7.

Online estimations of the parameters $\widehat{m}, \widehat{b}, \widehat{k}$, and $\widehat{k}_{p}$ are shown in Figure 8. Notice that, the effective estimation of the system parameters is achieved in a considerably short period of time (less than $200 \mathrm{~ms}$ ). For the case of the estimation of the viscous damping, there is no reference for comparison due to the ineffectiveness of the traditional identification methods when are applied to this particular system.

The comparison and results are summarized in Table 2.

The estimations are practically similar to the actual values. The average values of the real-time estimated parameters are $\widehat{m}=2.55 \mathrm{~kg}, \hat{k}=1290.34 \mathrm{~N} / \mathrm{m}$, and $\widehat{k}_{p}=-123.7 \times 10^{4} \mathrm{~N} / \mathrm{m}^{3}$, which are good approximations to the actual values in spite of inherent unmodelled dynamics and noisy measurements.

\subsection{Second Case Study: Two Degrees-of-Freedom Nonlinear} Vibrating System. A two-degrees-of-freedom configuration is now shown in Figure 9, where the nonlinear springs have a similar behavior to the one degree of freedom configuration.

The actual system parameters are reported in Table 3. Similarly, small viscous damping was neglected. For the evaluation of the nonlinearity index, based on the Hilbert transformation, we analyze the system response to the sinusoidal swept, where the amplitude $F$ is varied in the closed-time interval $[0.1,6.15] \mathrm{N}$, with 13 different measurements. In Figure 10, four measurements of the FRF and their corresponding Hilbert transformations in the Argand diagram are reported.

On the contrary, the nonlinearity index as a function of the input force amplitude is described in Figure 11. It can be confirmed that this system certainly exhibits high nonlinearities as far as the force input is increased. The corresponding Nyquist diagrams are shown in the right part of Figure 11. Here, we can observe a clear distortion on the Hilbert transforms for the original FRF (in blue), which is evident when the amplitude of the excitation force achieves a level of approximately $3 \mathrm{~N}$.

We also compute the nonlinearity index based on the Hilbert transform as defined in (8) and (9).

For the case of two degrees of freedom, we can apply the online algebraic identification approach as in the case of the single-degree-of-freedom system with some adaptations that are reported in [29]. First, we can describe the system dynamics by the set of coupled differential equations, when the force applied to the second mass carriage or degree of freedom is zero $\left(f_{2}(t) \equiv 0\right)$,

$$
\begin{gathered}
m_{1} x_{1}+b_{1} \dot{x}_{1}+k_{1} x_{1}+k_{p 1} x_{1}^{3}+b_{2}\left(\dot{x}_{1}-\dot{x}_{2}\right) \\
+k_{2}\left(x_{1}-x_{2}\right)-k_{p 2}\left(x_{2}-x_{1}\right)^{3}=f_{1}, \\
\frac{b_{2}}{m_{2}}\left(\dot{x}_{2}-\dot{x}_{1}\right)+\frac{k_{2}}{m_{2}}\left(x_{2}-x_{1}\right)+\frac{k_{p 2}}{m_{2}}\left(x_{2}-x_{1}\right)^{3}=-\ddot{x}_{2} .
\end{gathered}
$$

For the construction of the online and time-domain estimators, equations (20) and (21) are first multiplied by $(\Delta t)^{2}=\left(t-t_{0}\right)^{2}$ and then integrated by parts twice yielding 


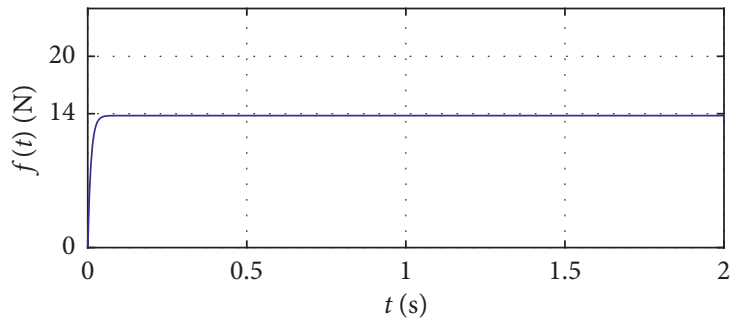

(a)

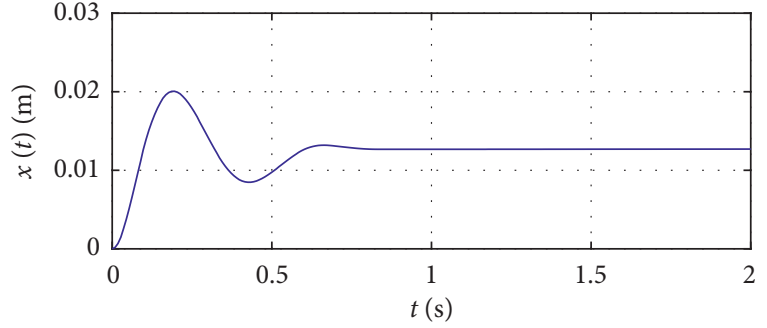

(b)

FIGURE 7: System response under step-type excitation: input force and transient response.

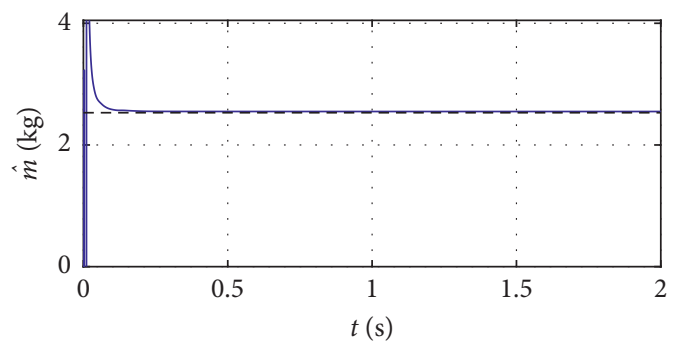

- Estimated

- - Actual

(a)

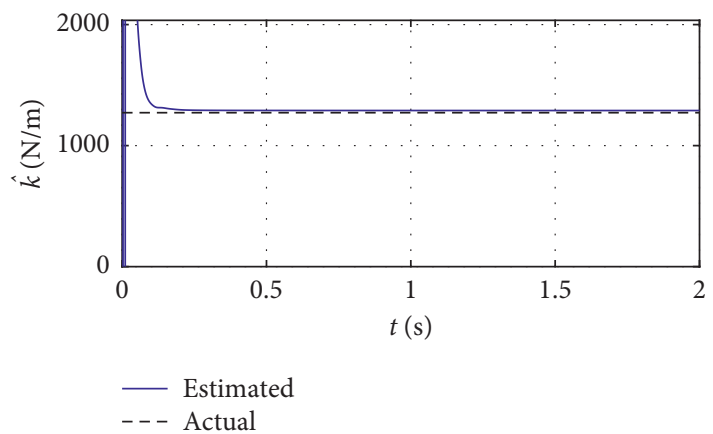

(c)

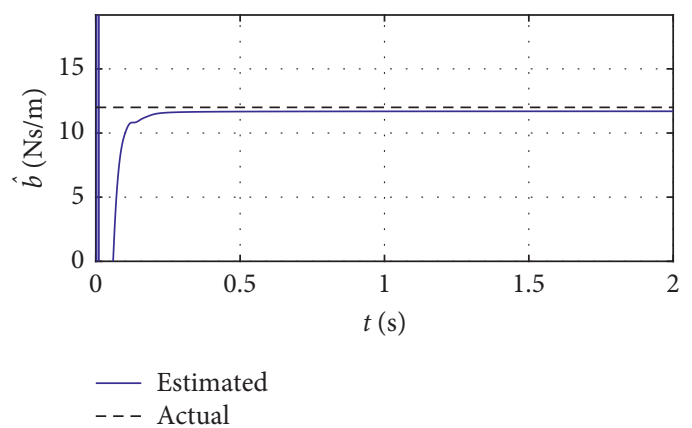

(b)

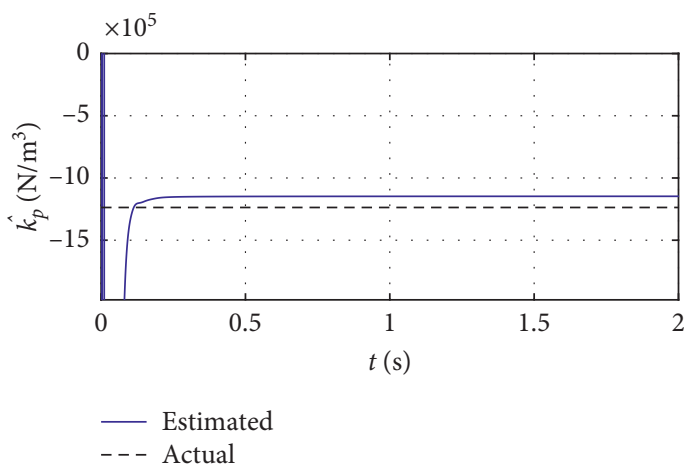

(d)

FIGURE 8: Online system parameters' identification.

$$
\begin{gathered}
m_{1}\left[2 \int_{t_{0}}^{(2)} x_{1}-4 \int_{t_{0}}(\Delta t) x_{1}+(\Delta t)^{2} x_{1}\right]+b_{1}\left[-2 \int_{t_{0}}^{(2)}(\Delta t) x_{1}+\int_{t_{0}}(\Delta t)^{2} x_{1}\right]+k_{1} \int_{t_{0}}^{(2)}(\Delta t)^{2} x_{1} \\
+k_{p 1} \int_{t_{0}}^{(2)}(\Delta t)^{2} x_{1}^{3}+b_{2}\left[-2 \int_{t_{0}}^{(2)}(\Delta t)\left(x_{1}-x_{2}\right)+\int_{t_{0}}(\Delta t)^{2}\left(x_{1}-x_{2}\right)\right]+k_{2} \int_{t_{0}}^{(2)}(\Delta t)^{2}\left(x_{1}-x_{2}\right) \\
-k_{p 2} \int_{t_{0}}^{(2)}(\Delta t)^{2}\left(x_{2}-x_{1}\right)^{3}=\int_{t_{0}}^{(2)}(\Delta t)^{2} f_{1}(t), \\
\frac{b_{2}}{m_{2}}\left[-2 \int_{t_{0}}^{(2)}(\Delta t)\left(x_{2}-x_{1}\right)+\int_{t_{0}}(\Delta t)^{2}\left(x_{2}-x_{1}\right)\right]+\frac{k_{2}}{m_{2}} \int_{t_{0}}^{(2)}(\Delta t)^{2}\left(x_{2}-x_{1}\right) \\
+\frac{k_{p 2}}{m_{2}} \int_{t_{0}}^{(2)}(\Delta t)^{2}\left(x_{2}-x_{1}\right)^{3}=\left[-2 \int_{t_{0}}^{(2)} x_{2}+4 \int_{t_{0}}(\Delta t) x_{2}-(\Delta t)^{2} x_{2}\right]
\end{gathered}
$$


TABle 2: Parameters' estimation summary.

\begin{tabular}{lccc}
\hline Parameter & Actual & Estimated & Difference (\%) \\
\hline$m \mathrm{~kg}$ & 2.53 & 2.55 & 0.79 \\
$b \mathrm{Ns} / \mathrm{m}$ & - & 12.47 & - \\
$k \mathrm{~N} / \mathrm{m}$ & 1272.1 & 1290.34 & 1.43 \\
$k_{p} \mathrm{Nm}^{-3}$ & $-123.7 \times 10^{4}$ & $-114.83 \times 10^{4}$ & 7.72 \\
\hline
\end{tabular}

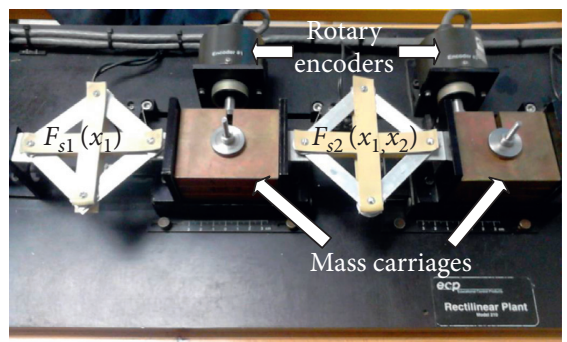

2 DoF non-linear experimental plant

(a)

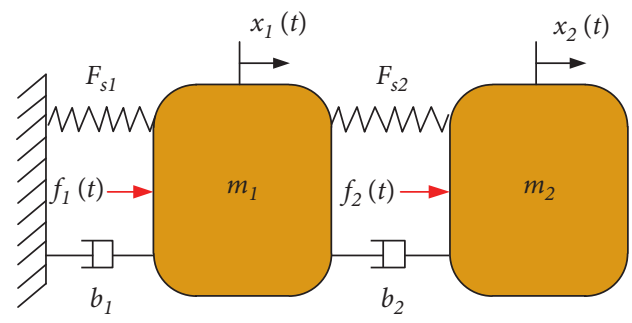

(b)

FIGURE 9: Two DOF vibrating mechanical system and its corresponding schematic representation.

TABLE 3: System parameters' 2DOF configuration.

\begin{tabular}{lcr}
\hline Parameter & First DOF & Second DOF \\
\hline$m$ & $2.35 \mathrm{~kg}$ & $2.754 \mathrm{~kg}$ \\
$b$ & - & - \\
$k$ & $1272.1 \mathrm{Nm}^{-1}$ & $1570 \mathrm{Nm}^{-1}$ \\
$k_{p}$ & $-1.237 \times 10^{6} \mathrm{Nm}^{-3}$ & $-1.350 \times 10^{6} \mathrm{Nm}^{-3}$ \\
\hline
\end{tabular}

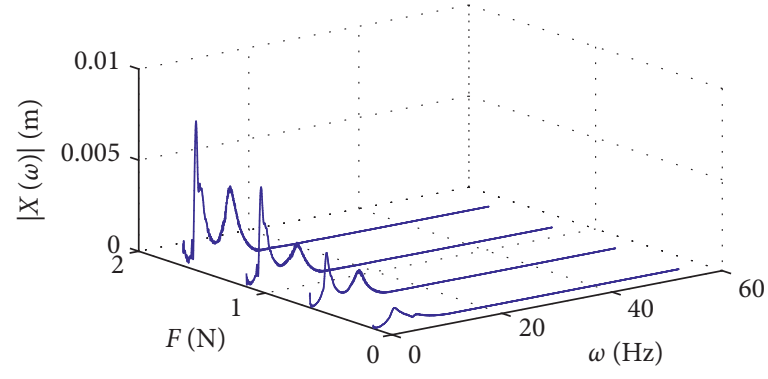

FIgure 10: Two-degrees-of-freedom case, system FRF at different excitation amplitudes.

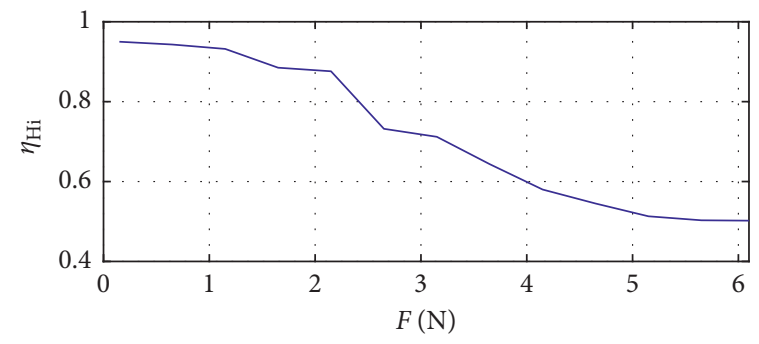

(a)

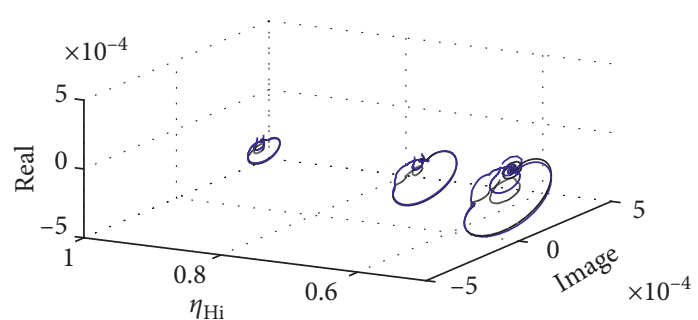

- Original FRF

- - - HT $\{$ FRF $\}$

FIGURE 11: Nonlinearity index based on Hilbert transformation and Hilbert transformation of FRF distortion. 
where $\int_{0}^{(n)} \phi(t)$ are iterated integrals such that: $\int_{t_{0}}^{t} \int_{t_{0}}^{\alpha_{1}} \ldots \int_{t_{0}}^{\alpha_{n-1}} \phi\left(\alpha_{n}\right) d \alpha_{n} \ldots d \alpha_{1}$, in the same way as the case of one degree of freedom. Here, we have expressions for the system parameters $m_{1}, b_{1}, b_{2}, k_{1}, k_{2}, k_{p 1}$, and $k_{p 2}$; these system parameters appear algebraically in equation (22). The identification of the system parameters for the case of two degrees of freedom is done by solving the algebraic equation (14) for each degree of freedom $x_{1}$ and $x_{2}$. Hence, the two independent algebraic equations for the system parameter identification are

$$
\begin{aligned}
& \mathbf{A}_{1} \theta_{1}=\mathbf{D}_{1}, \\
& \mathbf{A}_{2} \theta_{2}=\mathbf{D}_{2},
\end{aligned}
$$

where $\theta_{1}=\left[\widehat{m}_{1}, \widehat{b}_{1}, \widehat{k}_{1}, \widehat{k}_{p 1}, \widehat{b}_{2}, \widehat{k}_{2}, \widehat{k}_{p 2}\right]$ is the vector of the estimated parameters for the first degree of freedom $x_{1}$ and $\theta_{2}=\left[\widehat{b_{2} / m_{2}}, \widehat{k_{2} / m_{2}}, \widehat{k_{p 2} / m_{2}}\right]$ corresponds to the second degree of freedom $x_{2}$. Notice that, we cannot obtain the value of $m_{2}$ directly because of the zero force applied to the second degree of freedom; however, the value of the parameter $m_{2}$ is easily obtained by using the estimations of some of the two parameters calculated with equation (25). The matrices $\mathbf{A}_{\mathbf{1}}$, $\mathbf{D}_{1}, \mathbf{A}_{2}$, and $\mathbf{D}_{2}$ are, respectively, $7 \times 7,7 \times 1,3 \times 3$, and $3 \times 1$, given by

$$
\begin{aligned}
& \mathbf{A}_{1}=\left[\begin{array}{cccc}
a_{1(11)} & a_{1(12)} & \ldots & a_{1(17)} \\
a_{1(21)} & a_{1(22)} & \ldots & a_{1(27)} \\
\vdots & \vdots & \vdots & \\
a_{1(71)} & a_{1(72)} & \ldots & a_{1(77)}
\end{array}\right], \\
& \mathbf{D}_{1}=\left[\begin{array}{c}
d_{1(1)} \\
d_{1(2)} \\
\vdots \\
d_{1(7)}
\end{array}\right], \\
& \mathbf{A}_{2}=\left[\begin{array}{lll}
a_{2(11)} & a_{2(12)} & a_{2(13)} \\
a_{2(21)} & a_{2(22)} & a_{2(23)} \\
a_{2(31)} & a_{2(32)} & a_{2(33)}
\end{array}\right], \\
& \mathbf{D}_{2}=\left[\begin{array}{l}
d_{2(1)} \\
d_{2(2)} \\
d_{2(3)}
\end{array}\right] .
\end{aligned}
$$

The components $a_{1(i j)}$ and $d_{1(i)}$ for the matrix $A_{1}$ are

$$
\begin{aligned}
& a_{1(11)}=2 \int_{t_{0}}^{(2)} x_{1}-4 \int_{t_{0}}(\Delta t) x_{1}+(\Delta t)^{2} x_{1}, \\
& a_{1(12)}=-2 \int_{t_{0}}^{(2)}(\Delta t) x_{1}+\int_{t_{0}}(\Delta t)^{2} x_{1}, \\
& a_{1(13)}=\int_{t_{0}}^{(2)}(\Delta t)^{2} x_{1} \\
& a_{1(14)}=\int_{t_{0}}^{(2)}(\Delta t)^{2} x_{1}^{3}, \\
& a_{1(15)}=-2 \int_{t_{0}}^{(2)}(\Delta t)\left(x_{1}-x_{2}\right)+\int_{t_{0}}(\Delta t)^{2}\left(x_{1}-x_{2}\right), \\
& a_{1(16)}=\int_{t_{0}}^{(2)}(\Delta t)^{2}\left(x_{1}-x_{2}\right), \\
& a_{1(17)}=\int_{t_{0}}^{(2)}(\Delta t)^{2}\left(x_{2}-x_{1}\right)^{3}, \\
& d_{1(1)}=\int_{t_{0}}^{(2)}(\Delta t)^{2} f_{1} .
\end{aligned}
$$

The iterated integrations of equation (27) lead to the rest of the entries or components of the matrices $\mathbf{A}_{1}$ and $\mathbf{D}_{1}$ as follows:

$$
\begin{aligned}
& a_{1(k j)}=\int_{t_{0}} a_{1(k-1 j)}, \\
& d_{1(k)}=\int_{t_{0}} d_{1(k-1)},
\end{aligned}
$$

with $k=2, \ldots, 7$ and $j=1, \ldots, 7$. Likewise, the components $a_{2(i j)}$ and $d_{2(i)}$ for the matrix $A_{2}$ are

$$
\begin{aligned}
& a_{2(11)}=-2 \int_{t_{0}}^{(2)}(\Delta t)\left(x_{2}-x_{1}\right)+\int_{t_{0}}(\Delta t)^{2}\left(x_{2}-x_{1}\right), \\
& a_{2(12)}=\int_{t_{0}}^{(2)}(\Delta t)^{2}\left(x_{2}-x_{1}\right) \\
& a_{2(13)}=\int_{t_{0}}^{(2)}(\Delta t)^{2}\left(x_{2}-x_{1}\right)^{3} \\
& d_{2(1)}=-2 \int_{t_{0}}^{(2)} x_{2}+4 \int_{t_{0}}(\Delta t) x_{2}-(\Delta t)^{2} x_{2} .
\end{aligned}
$$

The iterated integrations of equation (29) lead to the rest of the entries or components of the matrices $\mathbf{A}_{2}$ and $\mathbf{D}_{2}$ as follows: 


$$
\begin{aligned}
& a_{2(k j)}=\int_{t_{0}} a_{2(k-1 j)}, \\
& d_{2(k)}=\int_{t_{0}} d_{2(k-1)},
\end{aligned}
$$

with $k=2,3$ and $j=1,2,3$. Hence, we have two independent algebraic expressions for the identification of the twodegrees-of-freedom nonlinear mechanical system:

$$
\begin{aligned}
& \theta_{1}=\left(\mathbf{A}_{1}\right)^{-1} \mathbf{D}_{1}=\frac{1}{\Delta_{1}}\left[\begin{array}{c}
\Delta_{1(1)} \\
\vdots \\
\Delta_{1(7)}
\end{array}\right], \\
& \theta_{2}=\left(\mathbf{A}_{2}\right)^{-1} \mathbf{D}_{2}=\frac{1}{\Delta_{2}}\left[\begin{array}{c}
\Delta_{2(1)} \\
\Delta_{2(2)} \\
\Delta_{2(3)}
\end{array}\right] .
\end{aligned}
$$

Then, the estimations of the system parameters $\widehat{m}_{2}, \widehat{b}_{1}$, $\widehat{b}_{2}, \widehat{k}_{1}, \widehat{k}_{2}, \widehat{k}_{p 1}$, and $\widehat{k}_{p 2}$ contained as components of the vectors $\theta_{1}$ and $\theta_{2}$ can be algebraically computed into some short window of time and without singularities by the estimators:

$$
\begin{aligned}
& \widehat{\theta}_{1}[i]=\operatorname{sgn}\left(\Delta_{1(i)}\right) \operatorname{sgn}\left(\Delta_{1}\right) \frac{\int_{t_{0}}^{(2)} e^{-\gamma\left(t-t_{0}\right)}\left|\Delta_{1(i)}\right|}{\int_{t_{0}}^{(2)} e^{-\gamma\left(t-t_{0}\right)}|\Delta|}, \quad i=1, \ldots, 7, \\
& \widehat{\theta}_{2}[i]=\operatorname{sgn}\left(\Delta_{2(i)}\right) \operatorname{sgn}\left(\Delta_{2}\right) \frac{\int_{t_{0}}^{(2)} e^{-\gamma\left(t-t_{0}\right)}\left|\Delta_{2(i)}\right|}{\int_{t_{0}}^{(2)} e^{-\gamma\left(t-t_{0}\right)}|\Delta|}, \quad i=1,2,3,
\end{aligned}
$$

where $\gamma \geq 0$ is an invariant filtering and smoothing gain. Here, - denotes estimate and "sgn" is the function defined in equation (2) which is only used to get the sign of the nonlinear stiffness parameters $\widehat{k}_{p 1}$ and $\widehat{k}_{p 2}$.

For experimental verification of the algebraic identification scheme, we use a step excitation force, applied to the first degree of freedom $x_{1}$ with an amplitude of 14 [N] and take measurements of the position of the two mass carriages $x_{1}$ and $x_{2}$ at a constant sampling period of 1 [ms]; both, the position signals $x_{1}(t)$ and $x_{2}(t)$ and the algebraic identification schemes (32) and (33) are obtained through a high-speed DSP board into a standard PC running under Windows $10^{\circledR}$ and Matlab ${ }^{\circledR} /$ Simulink $^{\circledR}$. The parameters of the mechanical system are reported in Table 1.

The performance of the algebraic identifiers (32) is shown in Figures 12 and 13 . Notice that it takes less than a half of a second to have stable and accurate estimations of the system parameters. For the case of estimator (33), the fast and accurate estimations of the normalized (respect to $m_{2}$ ) parameters are depicted in Figure 13.
The excitation force $f_{1}(t)$ and the system responses, $x_{1}(t)$ and $x_{2}(t)$, are shown in Figure 14 .

The results are summarized in Table 4 .

\section{Combination of Two Identification Techniques}

The proposed online algebraic system parameters' identification scheme is suitable to be implemented in complex digital systems based on microprocessors with x86 architecture, such as desktop personal computers and portable computers (laptops) running hard or soft real-time operating systems as verified, shown and proven in the previous sections of this work. Nowadays, it is common to use embedded digital systems that contain a digital signal processors working in conjunction with native peripherals such as analogue-to-digital converters (ADC modules) and direct memory access (DMA) for the complete implementation of the digital system, see [13].

In this section, we propose a combination of two methods for the algebraic identification of the parameters of the nonlinear system studied in this work. We propose the use of the orthogonal functions' signal approximation for the calculation of the iterated integrals involved in the online algebraic identification approach. On the contrary, by combining the technique reported in [22] and the proposed algebraic scheme [29], we have a contribution to the implementation of a system parameters' identification technique designed to be applied on embedded digital systems, completely based on matrix operations compatible with DSP libraries available in 32 bits ARM microcontrollers, see $[14,15]$. The flowchart of the process of system parameters' identification, for embedded systems, is depicted in Figure 15.

A set of functions are called orthogonal [22] in the interval $[a, b]$ if they satisfy

$$
\begin{array}{ll}
\int_{a}^{b} \phi_{m}(t) \phi_{k}(t)=0, & \text { if } m \neq k, \\
\int_{a}^{b} \phi_{m}(t) \phi_{k}(t)=\text { constant } \neq 0, & \text { if } m=k .
\end{array}
$$

It is well known that, in a certain interval, it is possible to approximate a given function by a finite sum of orthogonal functions. Consider the matrix form of the coupled equations (20) and (21) that describe the dynamics of the nonlinear mechanical systems shown in Figure 9:

$$
\mathbf{M} \ddot{\mathbf{x}}+\mathbf{B} \dot{\mathbf{x}}+\mathbf{K x}+\mathbf{K}_{\mathbf{p}} \mathbf{y}^{3}=\mathbf{f},
$$

were $\mathbf{M} \in R^{2 \times 2}, \quad \mathbf{B} \in R^{2 \times 2}$, and $\mathbf{K} \in R^{2 \times 2}$ are the mass, damping, and stiffness matrices, $\mathbf{K}_{\mathbf{p}} \in R^{2 \times 2}$ is the nonlinear stiffness matrix, the vector $\mathbf{x} \in R^{2}$ denotes the physical displacements of the mass carriages, and the vector $y \in R^{2}$ is defined as $\mathbf{y}=\left[\left(x_{2}-x_{1}\right),-x_{2}\right]^{T}$, with the vector $\mathbf{y}^{3}=\left[\left(x_{2}-x_{1}\right)^{3},-x_{2}^{3}\right]^{T}$. Finally, the exogenous force is represented in this particular experimental plant by the vector $\mathbf{f} \in R^{2}$ that, in this particular case, is $\mathbf{f}=\left[\begin{array}{ll}f(t) & 0\end{array}\right]$ 


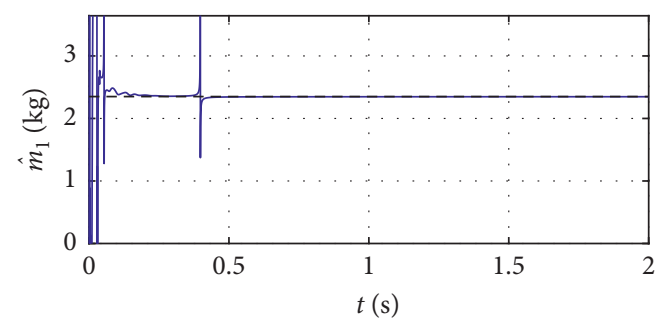

_ Estimated

- - - Actual

(a)

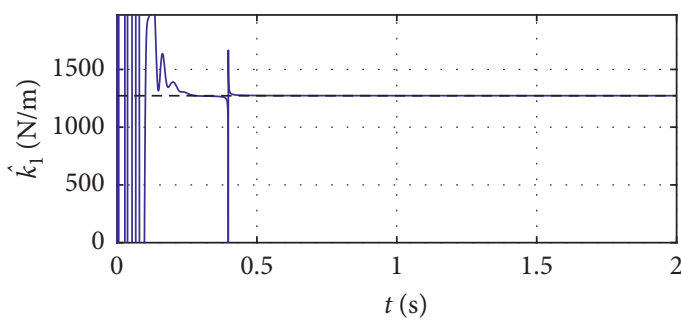

- Estimated

- - - Actual

(c)

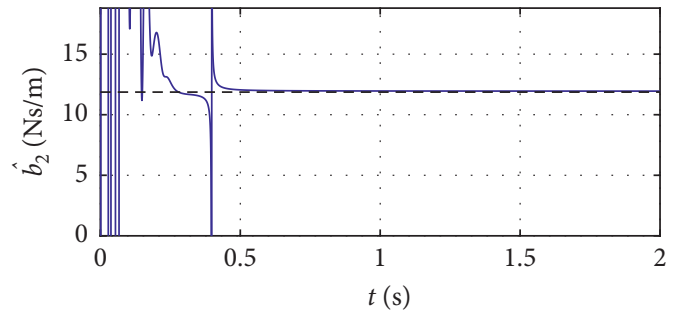

— Estimated

- - - Actual

(e)

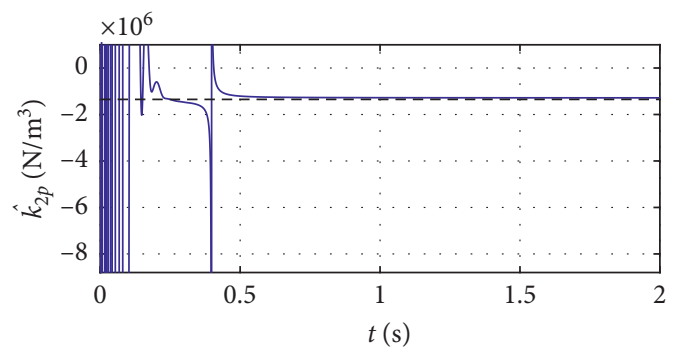

- Estimated

--- Actual

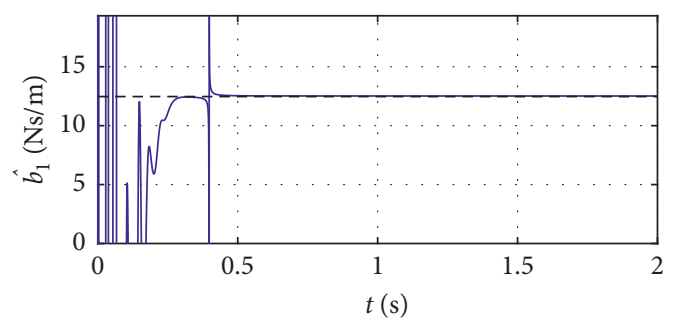

— Estimated

- - - Actual

(b)

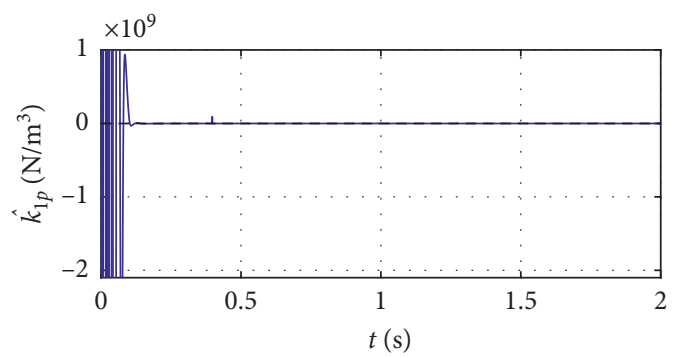

- Estimated

- - Actual

(d)

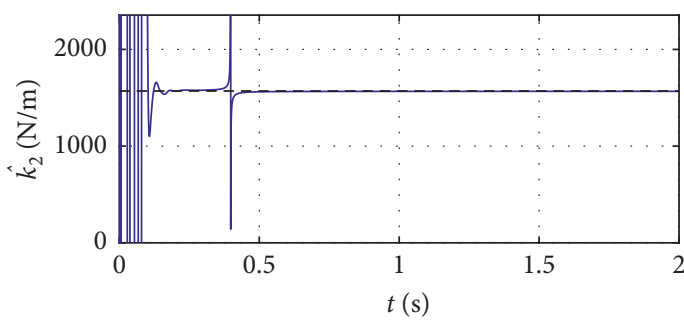

— Estimated

- - - Actual

(f)

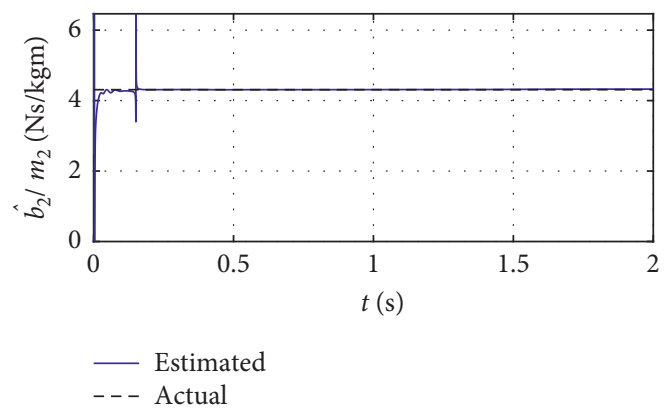

(h)

(g)

FIGURE 12: Online system parameters' identification 2DOF case. 


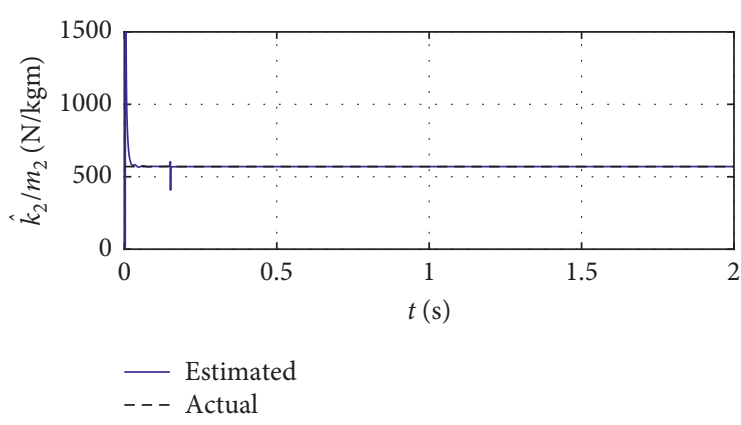

(a)

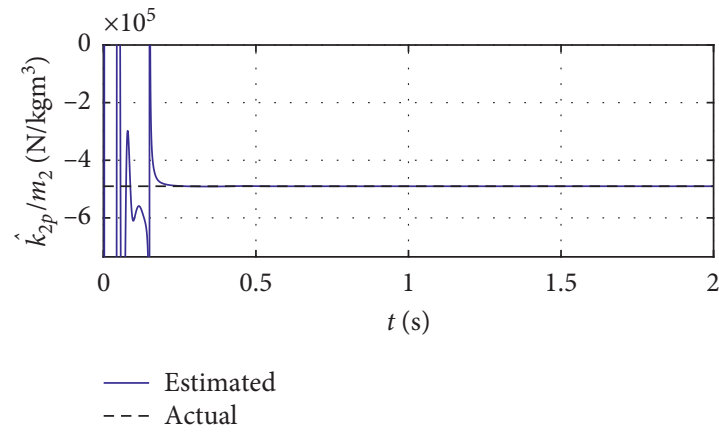

(b)

FIGURE 13: Online system parameters' identification 2DOF case, second degree of freedom.

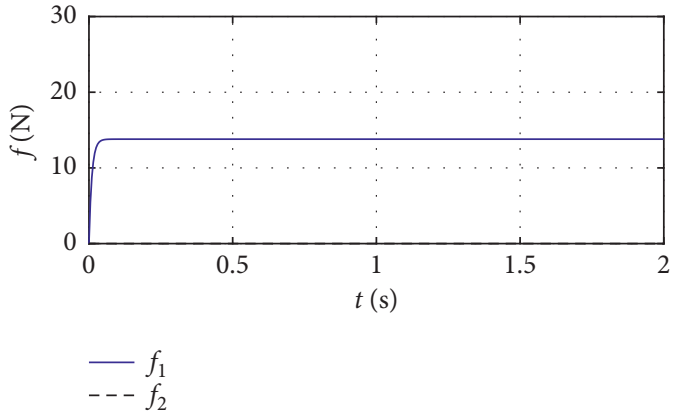

(a)

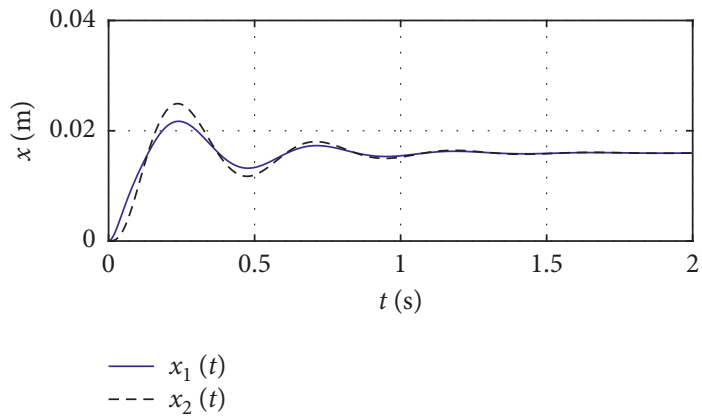

(b)

Figure 14: Two-degrees-of-freedom case.

TABLE 4: System parameters' estimation for the 2DOF configuration.

\begin{tabular}{lccc}
\hline Parameters & Actual & Estimated & Difference (\%) \\
\hline$m_{1}$ & $2.53 \mathrm{~kg}$ & $2.56 \mathrm{~kg}$ & 1.19 \\
$b_{1}$ & - & $12.48 \mathrm{Nsm}^{-1}$ & - \\
$k_{1}$ & $1272.1 \mathrm{Nm}^{-1}$ & $1280.7 \mathrm{Nm}^{-1}$ & 0.6 \\
$k_{p 1}$ & $-123.7 \times 10^{4} \mathrm{Nm}^{-3}$ & $-1304.7 \times 10^{4} \mathrm{Nm}^{-3}$ & 5.4 \\
$m_{2}$ & $2.754 \mathrm{~kg}^{2}$ & $2.80 \mathrm{~kg}$ & 1.6 \\
$b_{2}$ & - & $11.67 \mathrm{Ns}^{-1}$ & - \\
$k_{2}$ & $1570 \mathrm{Nm}^{-1}$ & $1585 \mathrm{Nm}^{-1}$ & 0.9 \\
$k_{p 2}$ & $-135.0 \times 10^{4} \mathrm{Nm}^{-3}$ & $-144.8 \times 10^{4} \mathrm{Nm}^{-3}$ & 7.2 \\
\hline
\end{tabular}

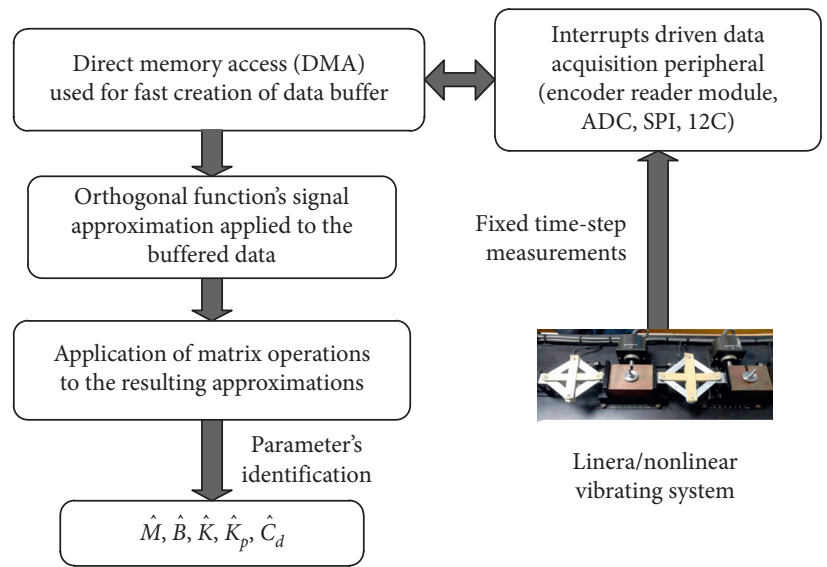

FIgURE 15: Flowchart of the combination of techniques as a buffered parameters' identification scheme. 
TABLE 5: System parameters' estimation, using orthogonal functions' signal approximation.

\begin{tabular}{lc}
\hline Term & Estimated \\
\hline$\overline{\mathbf{M}}$ & {$\left[\begin{array}{cc}2.57 & 0 \\
0 & 2.75\end{array}\right]$} \\
$\overline{\mathbf{B}}$ & {$\left[\begin{array}{cc}24.19 & -11.82 \\
-4.64 & 4.72\end{array}\right]$} \\
$\overline{\mathbf{K}}$ & {$\left[\begin{array}{cc}2848.7 & -1575.2 \\
-571.96 & -576.4\end{array}\right]$} \\
$\overline{\mathbf{K}}_{\mathbf{p}}$ & $1 \times 10^{6}\left[\begin{array}{cc}-1.23 & 1.355 \\
0 & -0.51\end{array}\right]$ \\
\hline
\end{tabular}

because the second degree of freedom is not actuated as described before. By dividing the second row of equation (36) by $m_{2}$, one obtains

$$
\overline{\mathbf{M}} \ddot{\mathbf{x}}+\overline{\mathbf{B}} \dot{\mathbf{x}}+\overline{\mathbf{K}} \mathbf{x}+\overline{\mathbf{K}_{\mathbf{p}}} \mathbf{y}^{3}=\overline{\mathbf{f}},
$$

where

$$
\begin{aligned}
& \overline{\mathbf{M}}=\left[\begin{array}{ll}
m_{1} & 0 \\
0 & 1
\end{array}\right], \\
& \overline{\mathbf{B}}=\left[\begin{array}{cc}
b_{1}+b_{2} & -b_{2} \\
-\frac{b_{2}}{m_{2}} & \frac{b_{2}}{m_{2}}
\end{array}\right] \text {, } \\
& \overline{\mathbf{K}}=\left[\begin{array}{cc}
k_{1}+k_{2} & -k_{2} \\
\frac{-k_{2}}{m_{2}} & \frac{k_{2}}{m_{2}}
\end{array}\right] \text {, } \\
& \overline{\mathbf{K}_{\mathbf{p}}}=\left[\begin{array}{cc}
k_{p 1} & -k_{p 2} \\
0 & \frac{k_{p 2}}{m_{2}}
\end{array}\right], \\
& \overline{\mathbf{f}}=\left[\begin{array}{c}
f(t) \\
-\ddot{x}_{2}
\end{array}\right] .
\end{aligned}
$$

Similarly, for the construction of the identifiers, by multiplying equation (37) by $(\Delta t)^{2}=\left(t-t_{0}\right)^{2}$ and integrating it by parts two times, we have

$$
\begin{aligned}
& \overline{\mathbf{M}}\left[2 \int_{t_{0}}^{(2)} \mathbf{x}-4 \int_{t_{0}}(\Delta t) \mathbf{x}+(\Delta t)^{2} \mathbf{x}\right] \\
& +\overline{\mathbf{B}}\left[-2 \int_{t_{0}}^{(2)}(\Delta t) \mathbf{x}+\int_{t_{0}}(\Delta t)^{2} \mathbf{x}\right] \\
& +\overline{\mathbf{K}} \int_{t_{0}}^{(2)}(\Delta t)^{2} \mathbf{x}+\overline{\mathbf{K}_{\mathbf{p}}} \int_{t_{0}}^{(2)}(\Delta t)^{2} \mathbf{y}^{3}=\int_{t_{0}}^{(2)}(\Delta t)^{2} \overline{\mathbf{f}}
\end{aligned}
$$

The orthogonal functions approximation of the integrands of equation (38), in the corresponding matrix representation by a sum of $r$ orthogonal functions, is then given by

$$
\begin{aligned}
\Delta t^{p} \mathbf{x} & \cong \mathbf{X}_{p,(1, r)} \phi(t)_{(1, r)}, \\
\Delta t^{2} \overline{\mathbf{f}} & \cong \mathbf{F}_{(1, r)} \phi(t)_{(1, r)}, \\
\Delta t^{2} \mathbf{y}^{3} & \cong \mathbf{Y}_{3,(1, r)} \phi(t)_{(1, r)},
\end{aligned}
$$

where $p=0,1,2$. Here, $\mathbf{X}, \mathbf{Y}_{3}$, and $\mathbf{F}$ are constant vectors with the coefficients of the orthogonal functions approximation of the integrands.

As reported in $[22,23]$, the orthogonal functions' signal approximation is useful in the solution of integral equations due to the property which allows to compute the iterated numerical integration as defined by the following matrix expression:

$$
\int_{t_{0}}^{(n)} \phi(\tau) \mathrm{d} \tau^{n} \cong \mathbf{P}^{n} \phi(t),
$$

where $\mathbf{P} \in R^{r \times r}$ is the so-called operational matrix of integration with constant elements, whose values depend on the orthogonal basis used. $\phi(t) \in R^{r}$ is a vector called the vectorial basis of the orthogonal series. In [23], a unified method for the operational matrix of integration computing is reported for the most popular orthogonal functions basis for signal approximation, and therefore, we can compute numerically the iterated integral using this property. The substitution of equations (39) in (38) yields to

$$
\begin{aligned}
& \overline{\mathbf{M}}\left[2 \mathbf{X}_{0} \phi(t) \mathbf{P}^{2}-4 \mathbf{X}_{1} \phi(t) \mathbf{P}+\mathbf{X}_{2} \phi(t)\right] \\
& +\overline{\mathbf{B}}\left[-2 \mathbf{X}_{1} \phi(t) \mathbf{P}^{2}+\mathbf{X}_{2} \phi(t) \mathbf{P}\right] \\
& +\overline{\mathbf{K}} \mathbf{X}_{2} \phi(t) \mathbf{P}^{2}+\overline{\mathbf{K}_{\mathbf{p}}} \mathbf{Y}_{3} \phi(t) \mathbf{P}^{2}=\mathbf{F} \phi(t) \mathbf{P}^{2}
\end{aligned}
$$

Since we use a given orthogonal basis $\phi(t)$, we can equate this coefficients so that using equations (39) and (40), we obtain the following matrix equation:

$$
\left[\overline{\mathbf{M}} \overline{\mathbf{B}} \overline{\mathbf{K}} \overline{\mathbf{K}}_{p}\right]\left[\begin{array}{c}
2 \mathbf{X}_{0} \mathbf{P}^{2}-4 \mathbf{X}_{1} \mathbf{P}+\mathbf{X}_{2} \\
-2 \mathbf{X}_{1} \mathbf{P}^{2}+\mathbf{X}_{2} \mathbf{P} \\
\mathbf{X}_{2} \mathbf{P}^{2} \\
\mathbf{Y}_{3} \mathbf{P}^{2}
\end{array}\right]=\left[\mathbf{F P}^{2}\right]
$$

Now, let us define

$$
\begin{aligned}
{[\boldsymbol{\Theta}]^{\mathbf{T}} } & =\left[\overline{\mathbf{M}} \overline{\mathbf{B}} \overline{\mathbf{K}} \overline{\mathbf{K}}_{p}\right], \\
{[\mathbf{A}] } & =\left[\begin{array}{c}
2 \mathbf{X}_{0} \mathbf{P}^{2}-4 \mathbf{X}_{1} \mathbf{P}+\mathbf{X}_{2} \\
-2 \mathbf{X}_{1} \mathbf{P}^{2}+\mathbf{X}_{2} \mathbf{P} \\
\mathbf{X}_{2} \mathbf{P}^{2} \\
\mathbf{Y}_{3} \mathbf{P}^{2}
\end{array}\right], \\
{[\mathbf{B}] } & =\left[\mathbf{F P}^{2}\right] .
\end{aligned}
$$

Thus, in a compact way, we can write

$$
[\boldsymbol{\Theta}]^{T}[\mathbf{A}]=[\mathbf{B}]
$$

This last expression constitutes the algebraic problem from identification, solved by using singular value decomposition, which allows to introduce the concept of 
pseudoinverse matrix, in order to solve the algebraic problem for the vector $[\Theta]$ as follows:

$$
[\boldsymbol{\Theta}]=[\mathbf{B}][\mathbf{A}]^{T}\left([\mathbf{A}][\mathbf{A}]^{T}\right)^{-1} .
$$

The results of the parameters' estimation when the excitation force is $\overline{\mathbf{f}}=\left[\begin{array}{ll}14 & 0\end{array}\right]^{T}[N]$ are reported in Table 5 .

\section{Conclusions}

In the present contribution, a time-domain algebraic scheme for online parameter estimation for an important class of nonlinear vibrating mechanical systems was introduced. Experimental results to prove the effectiveness of the parameter estimation using real-time position measurements were described. The satisfactory online fast estimation of the system parameters, performed in less than a second, was confirmed as well. Hence, results reveal that the algebraic nonlinear parametric estimation constitutes an excellent alternative with a superior performance to conventional identification techniques. Experimental system configurations involving nonlinear stiffness modelled such as algebraic polynomials were presented. The nonlinearity exhibited in experimental configurations was of the geometric type. The algebraic approach can be extended to other type of nonlinearities, such as Coulomb friction, as long as they appear in an algebraic form in the system structure. Moreover, two different identification methods, taking advantage of particular capabilities in both of them, were properly combined. Computationally speaking, we have improved the algebraic approach in structure; that is, iterated time integrations can be computed by using a compact and clear matrix expression, which is quite well defined and robust due to the good structure of the operational matrix of integration in an algebraic sense; the pseudoinverse is always possible to be computed and the results are obtained in finite time and well bounded to mention some of them. However, parameter estimations are performed slower like a price to pay to achieve stability on calculations. Furthermore, the presented nonlinearity indicator is easy to program and test. From this study, it is recommended to make a good analysis of numerical methods applied to original data in order to have a good criterion for the final determination of presence of nonlinearities. We have considered a reasonable value of $\eta \leq 0.9$ to establish that a given vibrating system exhibits relevant nonlinear oscillating dynamics. Otherwise, vibrating system dynamics could be represented in terms of a linear mathematical model, where small parametric nonlinearities can be considered such as unknown disturbances.

\section{Data Availability}

The data used to support the findings of the study are available from the corresponding author upon request.

\section{Conflicts of Interest}

The authors declare that they have no conflicts of interest.

\section{References}

[1] T. Söderström and P. Stoica, System Identification, PrenticeHall, Hoboken, NJ, USA, 1989.

[2] L. Ljung, System Identification: Theory for the User, PrenticeHall, Hoboken, NJ, USA, 1987.

[3] R. Isermann and M. Munchhof, Identification of Dynamic Systems: An Introduction with Applications, Springer-Verlag, Berlin, Germany, 2011.

[4] R. Manikantan, S. Chakraborty, T. K. Uchida, and C. P. Vyasarayani, "Parameter identification in nonlinear mechanical systems with noisy partial state measurement using pid-controller penalty functions," Mathematics, vol. 8, pp. 1281-1292, 2020.

[5] Y. Yi, S. Xing, and H. Yun, "Multidimensional system identification and active vibration control of a piezoelectric based sting system used in wind tunnel," Shock and Vibration, vol. 2020, Article ID 8856084, 15 pages, 2020.

[6] R. Shen, X. Qian, and J. Zhou, "Identification of linear nonviscous damping with different kernel functions in the time domain," Journal of Sound and Vibration, vol. 487, Article ID 115623, 2020.

[7] H. V. H. Ayala, D. Habineza, M. Rakotondrabe, and L. dos Santos Coelho, "Nonlinear black-box system identification through coevolutionary algorithms and radial basis function artificial neural networks," Applied Soft Computing, vol. 87, Article ID 105990, 2020.

[8] M. Feldman, Hilbert Transform Applications in Mechanical Vibration, John Wiley \& Sons, Chichester, UK, 2011.

[9] M. Feldman, "Non-linear system vibration analysis using Hilbert transform--I. Free vibration analysis method "Freevib"' Mechanical Systems and Signal Processing, vol. 8, no. 2, pp. 119-127, 1994.

[10] G. Golub and C. V. Loan, "An analysis of the total least squares problem," SIAM Journal on Numerical Analysis, vol. 17, no. 6, pp. 883-893, 1980.

[11] K. Worden and G. R. Tomlinson, Nonlinearity in Structural Dynamics: Detection, Identification and Modelling, Institute of Physics Publishing, Bristol, UK, 2001.

[12] G. R. Tomlinson, "Developments in the use of the hilbert transform for detecting and quantifying non-linearity associated with frequency response functions," Mechanical Systems and Signal Processing, vol. 1, no. 2, pp. 151-171, 1987.

[13] J. Yiu, The Definitive Guide to $A R M^{\circledR} C O R T E X^{\circledR}-M 3$ and CORTEX ${ }^{\circledR}-M 4$ Processors, Elsevier, Amsterdam, Netherlands, 2014.

[14] W. Mucha, "Real-time finite element simulations on arm microcontroller," Journal of Applied Mathematics and Computational Mechanics, vol. 16, no. 1, pp. 109-116, 2017.

[15] M. Alessandrini, G. Biagetti, P. Crippa, L. Falaschetti, L. Manoni, and C. Turchetti, "Singular value decomposition in embedded systems based on arm cortex-m architecture," Electronics, vol. 10, no. 1, 2020.

[16] F. Beltran-Carbajal and G. Silva-Navarro, "Output feedback dynamic control for trajectory tracking and vibration suppression," Applied Mathematical Modelling, vol. 79, pp. 793-808, 2020.

[17] O. Garcia-Perez, G. Silva-Navarro, and J. Peza-Solis, "Flexible-link robots with combined trajectory tracking and vibration control," Applied Mathematical Modelling, vol. 70, pp. 285-298, 2019.

[18] V. Ondra, I. A. Sever, and C. W. Schwingshackl, “A method for detection and characterisation of structural non-linearities 
using the hilbert transform and neural networks," Mechanical Systems and Signal Processing, vol. 83, pp. 210-227, 2017.

[19] S. F. Masri, J. Caffrey, T. K. Caughey, A. W. Smyth, and A. Chassiakosl, "Identification of the state equation in complex non-linear systems," International Journal of NonLinear Mechanics, vol. 39, pp. 111-1127, 2004.

[20] I. A. Kougioumtzoglou and P. D. Spanosl, "An identification approach for linear and nonlinear time-variant structural systems via harmonic wavelets," Mechanical Systems and Signal Processing, vol. 37, pp. 338-352, 2013.

[21] M. Fliess and H. Sira-Ramirez, "An algebraic framework for linear identification," ESAIM: Control, Optimization and Calculus of Variations, vol. 9, pp. 151-168, 2003.

[22] R. P. Pacheco and V. Steffen, "On the identification of nonlinear mechanical systems using orthogonal functions," International Journal of Non-Linear Mechanics, vol. 39, pp. 1147-1159, 2004.

[23] J. l. Wu, C. h. Chen, and C. f. Chen, "A unified derivation of operational matrices for integration in systems analysis, information technology," in Proceedings of the International Conference on Coding and Computing, pp. 436-442, Las Vegas, NV, USA, March 2000.

[24] I. Kovacic and M. J. Brennan, The Duffing Equation: Nonlinear Oscillators and Their Behaviour, John Wiley \& Sons, Hoboken, NJ, USA, 2011.

[25] G. R. Tomlinson and I. Ahmed, "Hilbert transform procedures for detecting and quantifying non-linearity in modal testing," Meccanica, vol. 22, no. 3, pp. 123-132, 1987.

[26] M. Simon and G. Tomlinson, "Use of the hilbert transform in modal analysis of linear and non-linear structures," Journal of Sound and Vibration, vol. 96, no. 4, pp. 421-436, 1984.

[27] J. N. Juang, Applied System Identification, Prentice-Hall, Hoboken, NJ, USA, 1994.

[28] L. G. Trujillo-Franco, G. Silva-Navarro, and F. Beltran-Carbajal, "Parameter estimation on nonlinear systems using orthogonal and algebraic techniques," in Proceedings of the Society for Experimental Mechanics Series, Society for Experimental Mechanics, pp. 347-354, Springer, Cham, Switzerland, April 2016.

[29] F. Beltran-Carbajal and G. Silva-Navarro, "Generalized nonlinear stiffness identification on controlled mechanical systems," Asian Journal of Control, vol. 21, no. 3, pp. 1281-1292, 2019.

[30] F. Beltran-Carbajal, G. Silva-Navarro, and L. G. TrujilloFranco, "A sequential algebraic parametric identification approach for nonlinear vibrating mechanical systems," Asian Journal of Control, vol. 19, no. 5, pp. 1-11, 2017. 